\title{
Different expression of cyclooxygenase-2 (COX-2) in selected nonmelanocytic human cutaneous lesions
}

\author{
Lukasz Kuźbicki ${ }^{1}$, Dariusz Lange ${ }^{2}$, Agata Stanek-Widera ${ }^{2}$, Barbara W. Chwirot ${ }^{1}$ \\ ${ }^{1}$ Department of Medical Biology, Institute of General and Molecular Biology, \\ Nicolaus Copernicus University, Torun, Poland \\ ${ }^{2}$ Department of Tumor Pathology, Maria Sklodowska-Curie Memorial Cancer Center \\ and Institute of Oncology, Gliwice, Poland
}

\begin{abstract}
The aim of our study was to elucidate the possible involvement of COX-2 in the development and/or progression of nonmelanocytic skin lesions. To evaluate the usefulness of that enzyme as a potential molecular marker, we examined the intensity and spatial distribution of COX-2 expression in selected types of such tumors using the same immunohistochemical procedure as in our earlier studies of melanocytic cancers. We examined 20 benign epithelial lesions, 11 precancerous lesions, 21 basal cell carcinomas (BCC), 14 squamous cell carcinomas (SCC) and eight fibromas. The levels of COX-2 expression detected in benign lesions and in normal skin were comparable. Elevated expression of this protein may play a role in the development of SCC, as indicated by strong immunostaining both in SCCs and precancerous lesions. Significantly stronger staining in SCCs compared to BCCs may indicate a role of COX-2 in cancer malignancy and serve as an indicator useful for differential diagnostics of the two types of cancer. Strong staining in all skin layers of SCC may help in detecting cancer cells infiltrating surrounding skin layers. (Folia Histochemica et Cytobiologica 2011; Vol. 49, No. 3, pp. 381-388)
\end{abstract}

Key words: cyclooxygenase-2 (COX-2), nonmelanocytic skin lesions, diagnostics, tumor invasiveness, immunohistochemistry

\section{Introduction}

Cyclooxygenases play a key role in the biosynthesis of prostaglandins [1,2]. Two isoforms of COX have been identified in human cells: COX-1 and COX-2, each encoded by a different gene [3, 4]. Constitutive expression of COX-1 has been found in cells in most human tissues $[1,3]$ while COX-2 is normally expressed only by cells of a few organs such as the brain, testes, trachea and kidney [5, 6]. COX-2 expression

Correspondence address: B.W. Chwirot, Department of Medical Biology, Institute of General and Molecular Biology, Nicolaus Copernicus University,

Gagarina Str. 9, 87-100 Torun, Poland;

tel.: (+ 48 56) 61145 99, fax: (+ 48 56) 61147 72;

e-mail: chwirot@biol.uni.torun.pl may also be induced in endothelial and smooth muscle cells and in fibroblasts after stimulation by growth factors, cytokines, lipopolysaccharides and tumor promoters [7, 8]. Many studies have demonstrated the overexpression of COX-2 in cells of different human tumors, mostly of epithelial origin like cancers of the colon and rectum [9-13], breast [14, 15], stomach $[16,17]$, lung [18], bladder [19-21], head and neck [22-24], ovary [25, 26], cervix [27, 28], prostate [29] and pancreas [30].

Human skin is composed of cells of different origin and thus the group of skin tumors is much differentiated. Our studies have demonstrated that the development and progression of human cutaneous melanoma are accompanied by a clear increase of COX-2 expression in melanocytes [31, 32]. It should be noted that earlier data on COX-2 expression in 
human cutaneous melanocytic lesions was very inconsistent. Recently, we have demonstrated that immunostaining with three different primary antibodies yielded significantly different percentage fractions of the COX-2 expressing melanocytes detected in individual naevi and melanomas, while the staining of normal skin was similar for all three antibodies [33].

Immunohistochemical studies of COX-2 expression in human cutaneous epithelial tumors and in normal skin reported to date have yielded quite inconsistent data. Leong et al. [34] found strong COX-2 expression in squamous cell carcinomas (SCC), while the cells of basal cell carcinomas (BCC) displayed a weak staining. The authors also detected the COX-2 expression of normal skin, but restricted to keratinocytes of the granular and spinous layers. Vogt et al. [35] were not able to detect the COX-2 protein in any of 11 cases of BCC, but observed medium and/or strong expression of the protein in nine of 17 cases of SCC and weak staining in two of six keratoacanthomas (KA). The staining pattern observed for cells of the normal skin was similar to that described by Leong et al. [34]. In the study by Putti et al. [36], COX-2 expression was detected in 13 of 17 SCCs and eight of 24 KAs. Kagoura et al. [37] examined 16 cases of $\mathrm{BCC}$ for the presence of the COX-2 protein and obtained immunostaining of six samples (two - strong, four - weak). The same authors reported slightly stronger COX-2 expression in ten of 13 cases of Bowen's disease (BD) and even stronger in 11 of 15 SCCs. In both types of the lesions, the percentage fractions of COX-2 positive cells in individual lesions varied significantly and metastases expressed COX-2 at lower levels than primary SCC and BD. In normal skin, the COX-2 expression was found only in differentiated keratinocytes, similarly to observations of Leong et al. [34] and Vogt et al. [35]. Nijsten et al. [38] described comparable levels of COX-2 expression in SCCs and actinic keratoses (AK), stronger than in BDs, while in normal skin they were able to detect COX-2 in only two of 35 cases investigated with the staining limited to cells of the granular layer. Interestingly, several authors did not detect COX-2 expression in keratinocytes of granular and spinous layers and instead reported the staining of cells within the basal layer [39-43]. Müller-Decker et al. [39] observed COX-2 staining of some cells of the epidermal basal layer in only two of nine samples of normal skin. In the same study, clear COX-2 expression was detected in all examined cases of AK, KA, SCC and in one case described as skin cancer in situ. Weak expression of COX-2 was also demonstrated in six of eight BCCs. COX-2 positive cells were present in SCCs in all the skin layers, but the strongest expression was found in the spinous layer of cancer in situ. Kim et al. [40] were able to detect COX-2 expression in only five of ten SCCs, four of ten cases of BD, five of ten AKs and two of ten cases of porokeratosis. At the same time the authors detected COX-2 in eight of ten BCCs. Some of the COX-2 positive lesions displayed a strong staining: $\mathrm{BD}-50 \%, \mathrm{AK}-20 \%$ and BCC $-12.5 \%$ of positive cases. In normal skin, a weak staining was found in the epidermal basal layer in four of ten specimens. Finally, An et al. [41] did not find any COX-2 expression in normal human skin but the COX-2 positive cells were detected both within the lesions and/or in stroma for ten of 11 SCCs, seven of eight cases of BD, ten of 12 AKs and nine of 16 BCCs. Characteristically, the COX-2 positive cells of $\mathrm{BD}$ and $\mathrm{SCC}$ were always present in epidermal layers above the basal layer, while BCCs generally displayed positive staining of stromal cells. Only in two cases of BCC were the positive cells present both within the lesions and the stroma.

In this study, we examined specimens of selected types of nonmelanocytic cutaneous lesions (benign and precancerous epithelial lesions, SCCs and BCCs) for differences in both COX-2 expression levels and spatial distributions of the COX-2 positive cells. Immunohistochemical detection of the COX-2 protein was carried out using the procedure elaborated earlier for studies of melanocytic lesions of human skin.

\section{Material and methods}

Patient material. Formalin-fixed paraffin-embedded tissue samples were obtained from pathology units of the Municipal and Regional Hospitals, Torun, Poland. The study was carried out as part of a project approved by the Committee of Scientific Research at the School of Medicine, Bydgoszcz, Poland. The samples were characterized independently by the hospital staff and by pathologists from the Department of Tumor Pathology, Oncology Centre - Maria Sklodowska-Curie Institute, Gliwice, Poland. The material included 20 benign epithelial lesions (verruca seborrhoica - 14, papilloma - six), 11 precancerous lesions (AK - two, KA three, BD - six) and 35 malignant lesions (BCC - 21, SCC - 14). The group of the BCCs included five superficial, 11 nodular, two adnexal, one cystic and two mixed (nodular and infiltrating) types. The group of the SCCs could be stratified according to the differentiation degree into six cases of high, three of medium and one of low degree of differentiation. Eight fibromas were also involved in the study.

Immunohistochemistry. The procedure of immunohistochemical staining, elaborated and tested using samples of normal human skin, was described in our earlier papers on COX-2 detection in human cutaneous melanocytic lesions 
[31, 32]. Briefly, $3 \mu \mathrm{m}$ sections were mounted on 3-aminopropyltriethoxysilane (APES; Sigma-Aldrich, St. Louis, MI, USA), dewaxed and rehydrated. The slides were then immersed in $0.01 \mathrm{M}$ citrate buffer $\mathrm{pH} 6.0$ and heated for 60 $\min$ at $90^{\circ} \mathrm{C}$. Endogenous peroxidase was blocked by $30 \mathrm{~min}$ incubation with $0.3 \% \mathrm{H}_{2} \mathrm{O}_{2}$ (Sigma-Aldrich) and nonspecific binding of antibodies was prevented by $30 \mathrm{~min}$ incubation with $1.5 \%$ normal rabbit serum (Vector Laboratories, Burlingame, CA, USA). Finally, the slides were incubated in a humid chamber at room temperature for $60 \mathrm{~min}$ with primary goat polyclonal COX-2 antibody (Santa Cruz Biotechnology, Santa Cruz, CA, USA) in a dilution 1:100 v/v in $\mathrm{PBS} \mathrm{pH}$ 7.4. Immunocomplexes were visualized using the peroxidase technique with Vectastain Elite ABC Kit (Vector Laboratories) according to the procedure supplied with the kit, and stained using $0.05 \%$ 3.3'-diaminobenzidine (DAB; Sigma-Aldrich) with $0.01 \% \mathrm{H}_{2} \mathrm{O}_{2}$ (Sigma-Aldrich) and $0.06 \% \mathrm{NiCl}_{2}$ (Sigma-Aldrich). Owing to the inclusion of $\mathrm{NiCl}_{2}$ the color of the reaction product changed from typical brown to blue to black [44, 45], making the precipitate easier to detect against the background of melanin present in some of the lesions investigated. Negative control sections were incubated with PBS pH 7.4 solution without primary antibody and did not display any reaction products.

Microscopic examination was done using a BX41 microscope (Olympus Optical Co., Tokyo, Japan) with white light illumination. Digital images were acquired with a ColorView III camera (Soft Imaging System, Hanover, Germany) and analySIS 3.2 software (Soft Imaging System).

Quantitative immunohistochemical scoring involved determination of percentage fractions of the COX-2 positive cells. Cell counts were carried out for individual skin layers for three fields of view at magnification $\times 1,000$. Three regions were analyzed for each section: the center and periphery of the lesion and adjacent normal skin. The intensity of immunostaining of individual cells was also determined and classified as weak (blue, + ) or strong (dark blue to black, ++ ).

Statistical analysis. Data (percentage fractions of the positively stained cells) is presented as mean values \pm standard deviations calculated for the central (c) and border (b) regions of the lesions and for the adjacent normal skin. Statistical significance of the data was assessed using Student's $t$-test with the standard $\mathrm{p}<0.05$ condition (Microsoft Excel; Microsoft, Redmond, WA, USA).

\section{Results}

We investigated the intensity and spatial pattern of the expression of COX-2 in 74 nonmelanocytic skin lesions and in the adjacent normal skin. Immunoreactivity of the COX-2 protein showed cytoplasmic, often perinuclear, staining in both tumor and normal skin cells.

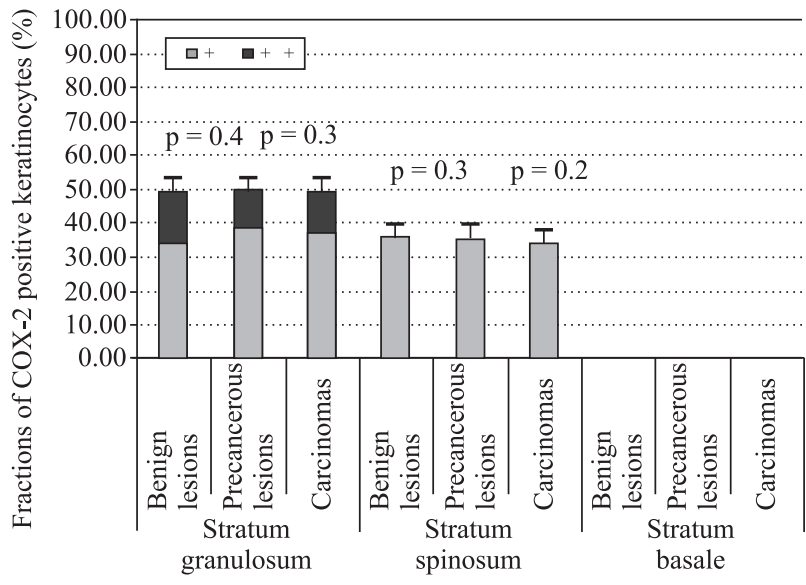

Figure 1. Mean percentage fractions of cyclooxygenase-2 (COX-2) positive keratinocytes in normal skin adjacent to benign, precancerous and malignant lesions. Dark-shaded and light-shaded fields illustrate the contribution of cells with heavy $(++)$ and light $(+)$ staining, respectively

Normal skin adjacent to the lesions showed characteristic pattern of positive staining of keratinocytes of the spinous and granular layers. The staining intensity increased towards the skin surface and was slightly stronger in the granular layer than in the spinous layer. No expression of COX-2 was observed in the basal layer. COX-2 expression and the spatial distribution of the COX-2 positive keratinocytes were similar in normal skin surrounding skin lesions of different types i.e. benign, precancerous and malignant (Figures 1,2A).

The COX-2 expression in benign epithelial skin lesions (verrucas and papillomas) was similar to that in the normal skin (Figures 1, 2A, B, 3A). The staining pattern was the same for central and peripheral regions of the lesions.

All three types of precancerous lesion (AK, KA and BD) expressed COX-2 significantly more strongly than the benign lesions, both in terms of percentage fractions of the COX-2 positive cells and of the contribution of cells with a strong $(++)$ staining (Figures 2C, 3B). Enhanced COX-2 expression was observed in the spinous and granular layers of the epidermis. Moreover, unlike the normal skin and benign lesions, the precancerous lesions also showed staining of keratinocytes of the epidermal basal layer, although the percentage fraction of the COX-2 positive cells was in this layer significantly lower compared to the spinous layer (Figure 3C).

BCCs expressed COX-2 weakly at levels lower than the benign epithelial lesions $\left(\mathrm{p}_{\mathrm{c}}=0.02, \mathrm{p}_{\mathrm{b}}=0.01\right)$. Percentage fractions of the COX-2 positive cells were similar (within limits of standard deviations) in all the histologic types of BCC investigated (Figure 3D). The highest fractions of cells with positive staining were 

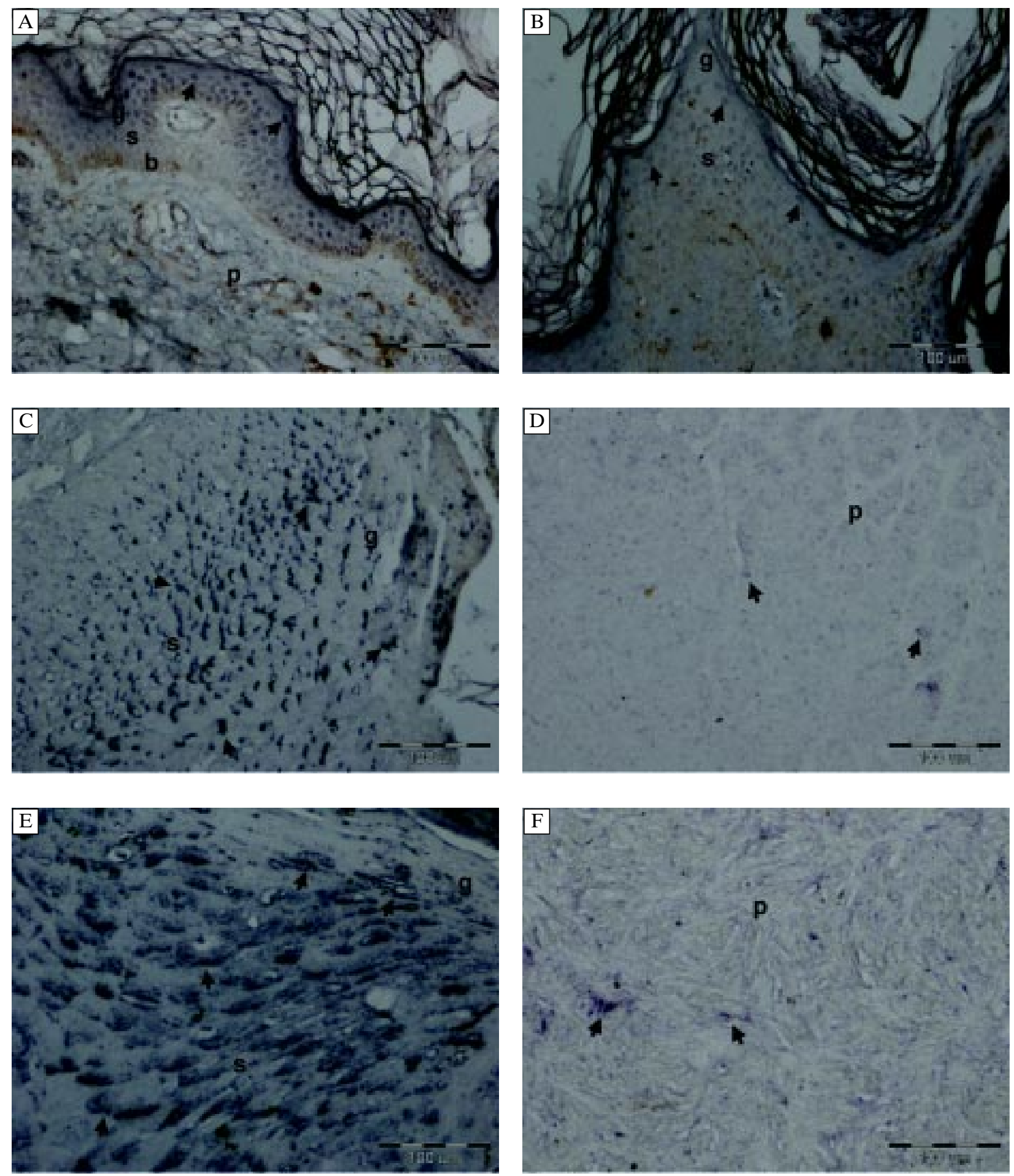

Figure 2. Immunoreactivity for cyclooxygenase-2 (COX-2) in: normal skin (A), verruca seborrhoica (B), AK (C), BCC (D), SCC (E) and fibroma (F). Respective images illustrate areas of central regions of the lesions. Different layers of the skin designated as follows: $\mathrm{b}$ - basal; $\mathrm{g}$ - granular; $\mathrm{p}$ - papillary; $\mathrm{r}$ - reticular; $\mathrm{s}$ - spinous; COX-2 positive cells indicated by arrows; magnification $\times 200$

consistently found in the granular layer, slightly lower in the spinous layer, and significantly lower in deeper layers of the skin. The COX-2 positive cells present in the epidermal basal layer and in the dermis were not only significantly less numerous but also less often demonstrated strong $(++)$ staining (Figures 2D, 3E).

Unlike the BCCs, all the SCC lesions showed very strong COX-2 expression, comparable to the expression detected in the precancerous lesions $\left(\mathrm{p}_{\mathrm{c}}=0.3\right.$, 

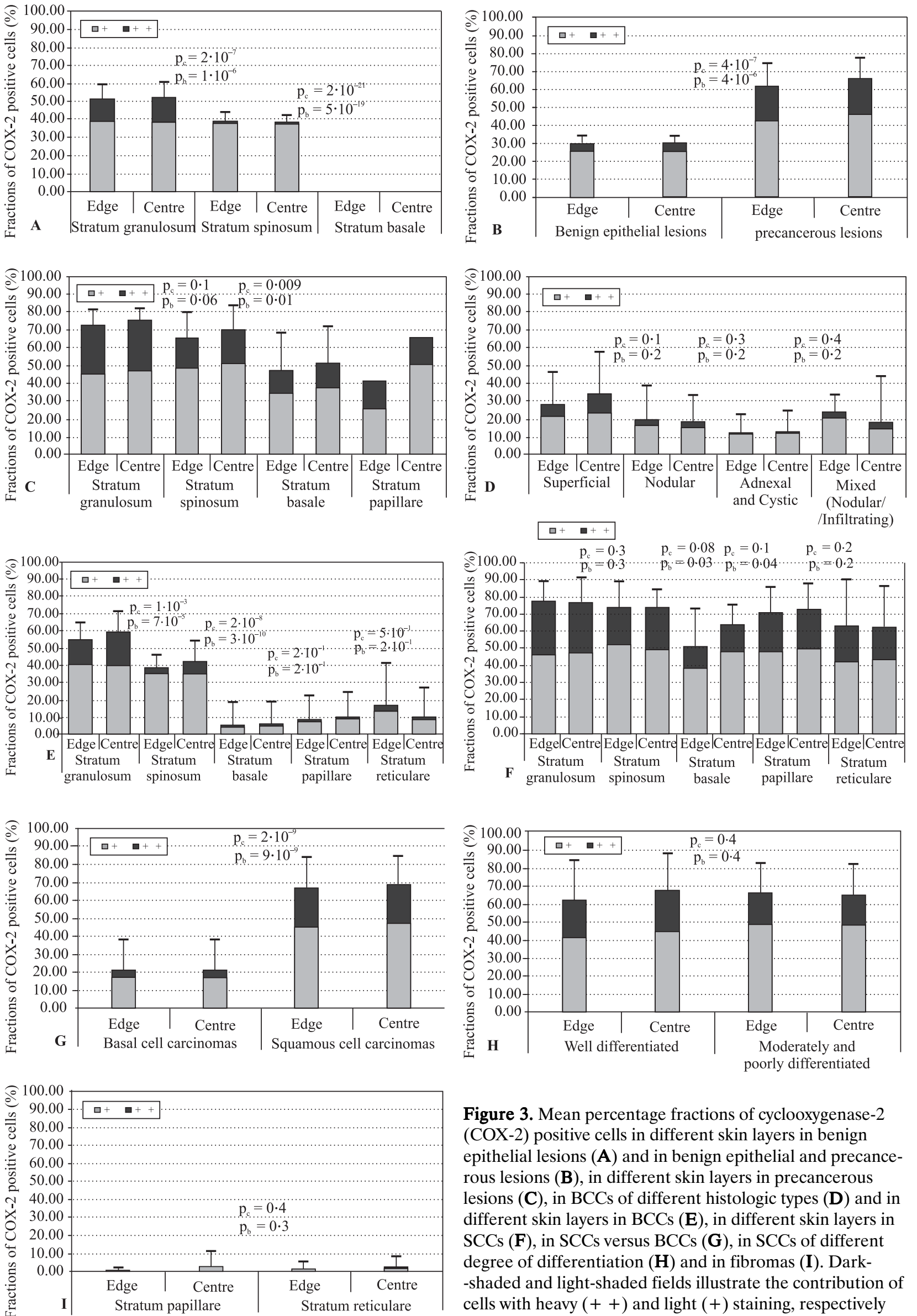

Figure 3. Mean percentage fractions of cyclooxygenase-2 (COX-2) positive cells in different skin layers in benign epithelial lesions (A) and in benign epithelial and precancerous lesions (B), in different skin layers in precancerous lesions (C), in BCCs of different histologic types (D) and in different skin layers in BCCs $(E)$, in different skin layers in SCCs $(\mathbf{F})$, in SCCs versus BCCs $(\mathbf{G})$, in SCCs of different degree of differentiation $(\mathbf{H})$ and in fibromas $(\mathbf{I})$. Dark-shaded and light-shaded fields illustrate the contribution of cells with heavy $(++)$ and light $(+)$ staining, respectively 
$\mathrm{p}_{\mathrm{b}}=0.2$; compare Figures $\left.2 \mathrm{C}, \mathrm{E}\right)$. The only exception was the slightly lower expression of COX-2 protein by the SCC cells present in the basal layer at peripheral regions of the lesions (Figure 3F). Both the percentage fraction of COX-2 positive cells and the frequency of such cells displaying strong $(++)$ staining were higher in the SCC group than in the benign lesions $\left(\mathrm{p}_{\mathrm{c}}=2 \cdot 10^{-7}, \mathrm{p}_{\mathrm{b}}=8 \cdot 10^{-7}\right.$; Figures $\left.2 \mathrm{~B}, \mathrm{E}\right)$ or in the BCCs (Figures 2D, E, 3G) and the differences were statistically significant. No differences were found between SCCs of different grades of differentiation (Figure $3 \mathrm{H}$ ). Also the cancer cells present in different skin layers showed similar levels and patterns of COX-2 expression.

Fibromas expressed COX-2 at very low or undetectable levels (Figures 2F, 3I). Normal skin surrounding fibromas showed a staining pattern similar to that of normal skin adjacent to epithelial lesions.

Finally, there was no difference in the data obtained for central and peripheral regions of the lesions.

\section{Discussion}

Despite 15 years of study, neither the role nor the patterns of COX-2 expression in normal human skin and cutaneous pathologic lesions have yet been fully elucidated. Literature data on COX-2 expression in normal skin varies from reports of nondetectable expression levels [41], to the expression observed only in some cells of the granular and spinous layers of the epidermis $[34,35,37]$ or restricted exclusively to the epidermal basal layer [39, 40, 42, 43]. Leong et al. [34] described an association between differentiation of human keratinocytes in vitro and expression of COX-2. Well known possible sources of discrepancies in the reported results may include differences in antibodies used, preparation of the material from fixation and embedding to antigen retrieval, etc.

In this work, and in our earlier studies of COX-2 in melanocytic cutaneous lesions and in adjacent skin $[31,32]$, we have consistently observed in a large number of samples of normal human skin positive staining of keratinocytes of the granular and spinous layers. Our present study demonstrated that papillomas, verrucas and normal skin express COX-2 at comparable levels. Thus, development of these benign lesions is not associated with enhanced expression of COX-2 by keratinocytes.

Fibroblasts of normal skin did not express COX-2 at a detectable level. On the other hand, fibromas were characterized by very weak staining of fibroblasts. Adler et al. [46] also reported COX-2 expression in 19 of 20 fibromas; and in $75 \%$ of the cases, the fraction of stained cells exceeded $34 \%$.
Precancerous lesions investigated in this study (AK, KA, BD) expressed COX-2 significantly more strongly than the benign epithelial lesions. Interestingly, elevated expression of the enzyme was observed not only in the granular and spinous layers but also in the basal layer which did not show any staining in the normal skin and the benign lesions. Epidemiological data indicates a strong relation between the presence of AK and the development of SCC: $82.4 \%$ of such skin cancers were found either within $(26.7 \%)$ or close to $(55.7 \%)$ actinic keratoses [47]. The estimated ten-year risk of developing SCC against a background of AK is 13-20\% [48], while for KA the risk of progression to SCC is 3.6-13.9\%, depending on the age group [49]. In our study, the precancerous lesions and SCCs expressed COX-2 at a similar level. Although some authors have reported stronger COX-2 staining in SCCs compared to KA [35, 36] more groups have described similar (or only slightly higher) COX-2 expression in SCCs compared to precancerous lesions like AK, KA and BD [37-41].

It is thus plausible that elevated COX-2 expression plays a role in the development of SCC. Such a conclusion is further supported by the therapeutic efficacy of diclofenac (COX-2 inhibitor) in the treatment of AK [50].

BCCs examined in this work showed a weak expression of COX-2, comparable with, or lower than, that observed for the benign epithelial lesions. Such a result is in general agreement with earlier reports of other groups which have described very low COX-2 expression in most BCCs (undetectable in others) $[39,40]$ or undetectable COX-2 expression in the majority of cases with weak expression found only in a small fraction of the lesions investigated [34, 35, 37, 41]. Taking into account the good reproducibility of our results for a large number of samples of normal skin, and the consistency of the results obtained for BCCs, it seems reasonable to conclude that the reports of undetectable COX-2 expression in smaller or larger fractions of the BCCs examined in earlier studies reflected insufficient sensitivity of the COX-2 detection rather than a lack of the protein in the BCC cells.

The SCCs investigated in this present work expressed COX-2 significantly more strongly than the BCCs. A similar relation has been found in several earlier studies [34, 35, 37, 39-41]. Differences in both the level and pattern of COX-2 expression by cells of the two types of skin cancer may be due to the fact that SCC originates from more differentiated cells and COX-2 has been suggested to be involved in the differentiation of keratinocytes [34]. Furthermore, the observation that all the SCCs show a similarly enhanced expression of COX-2 independently of the 
differentiation degree may indicate a role of COX-2 in the development of SCC, but not necessarily in the progression of the disease.

Strong staining of the SCC cells present in any of the skin layers makes the COX-2 protein a molecular marker useful for precise and sensitive detection of the cancer cells in histopathologic specimens.

The staining pattern observed for SCCs is very different from BCC staining, which is typically limited to a zone of differentiated skin cells with a very weak staining of the cancer cells present in the basal layer and dermis. The staining pattern of BCCs was similar for all the histologic types of the lesions and the expression level did not change even at stages of local invasiveness.

In our earlier study [51], we also observed strong expression of cyclin-dependent kinase 2 (CDK-2) of the SCC cells across all the skin layers. Similarity of the expression patterns of CDK-2 and COX-2 in SCCs may indicate a role of elevated COX-2 expression in the proliferation of cancer cells. Such a conclusion is further supported by in vitro studies by Higashi et al. [52] who found that inhibiting COX-2 expression caused growth suppression of the SCC cells.

In conclusion, our study demonstrates that neither the development of benign epithelial skin lesions and BCC, nor the local invasiveness of the latter, are associated with enhanced COX-2 expression. An elevated level of this enzyme seems to play a role in the development of SCC. This is evidenced by strong staining found both for the cancerous and precancerous lesions. Significantly higher COX-2 expression in SCC compared to BCC may be useful for differential diagnostics of the two cancer types, while strong staining of the SCCs across all the skin layers may help in detecting cancer cells in histopathologic examinations.

\section{Acknowledgement}

This work was supported by Polish state budget funds for research in the years 2010-2011.

\section{References}

1. Kurumbail RG, Kiefer JR, Marnett LJ. Cyclooxygenase enzymes: catalysis and inhibition. Curr Opin Struct Biol. 2001;11:752-760.

2. Subbaramaiah K, Dannenberg AJ. Cyclooxygenase 2: a molecular target for cancer prevention and treatment. Trends Pharmacol Sci. 2003;24:96-102.

3. Morita I. Distinct functions of COX-1 and COX-2. Prostaglandins Other Lipid Mediat. 2002;68-69:165-175.

4. Tanabe T, Tohnai N. Cyclooxygenase isozymes and their gene structures and expression. Prostaglandins Other Lipid Mediat. 2002;68-69:95-114.

5. Smith WL, Garavito RM, DeWitt DL. Prostaglandin endoperoxide H synthases (cyclooxygenases)-1 and -2. J Biol Chem. 1996;271:33157-33160.
6. Harris RC. Cyclooxygenase-2 in the kidney. J Am Soc Nephrol. 2000;11:2387-2394.

7. Singh B, Lucci A. Role of cyclooxygenase- 2 in breast cancer. J Surg Res. 2002;108:173-179.

8. Trifan OC, Hla T. Cyclooxygenase- 2 modulates cellular growth and promotes tumorigenesis. JCell Mol Med. 2003;7: 207-222.

9. Yamauchi T, Watanabe M, Kubota T et al. Cyclooxygenase-2 expression as a new marker for patients with colorectal cancer. Dis Colon Rectum. 2002;45:98-103.

10. Chapple KS, Cartwright EJ, Hawcroft G et al. Localization of cyclooxygenase-2 in human sporadic colorectal adenomas. Am J Pathol. 2000;156:545-553.

11. Knösel T, Yu Y, Stein U et al. Overexpression of cyclooxygenase- 2 correlates with chromosomal gain at the cyclooxygenase-2 locus and decreased patient survival in advanced colorectal carcinomas. Dis Colon Rectum. 2004;47:70-77.

12. Tomozawa S, Tsuno NH, Sunami E et al. Cyclooxygenase-2 overexpression correlates with tumour recurrence, especially haematogenous metastasis, of colorectal cancer. Br J Cancer. 2000;83:324-328.

13. Kakiuchi Y, Tsuji S, Tsujii M et al. Cyclooxygenase-2 activity altered the cell-surface carbohydrate antigens on colon cancer cells and enhanced liver metastasis. Cancer Res. 2002;62:1567-1572.

14. Ristimäki A, Sivula A, Lundin J et al. Prognostic significance of elevated cyclooxygenase-2 expression in breast cancer. Cancer Res. 2002;62:632-635.

15. Boland GP, Butt IS, Prasad R, Knox WF, Bundred NJ. COX-2 expression is associated with an aggressive phenotype in ductal carcinoma in situ. Br J Cancer. 2004;90:423-429.

16. Lim HY, Joo HJ, Choi JH et al. Increased expression of cyclooxygenase-2 protein in human gastric carcinoma. Clin Cancer Res. 2000;6:519-525.

17. Yu LZ, Gao HJ, Bai JF et al. Expression of COX-2 proteins in gastric mucosal lesions. World J Gastroenterol. 2004;10: 292-294.

18. Fang HY, Lin TS, Lin JP, Wu YC, Chow KC, Wang LS. Cyclooxygenase-2 in human non-small cell lung cancer. Eur J Surg Oncol. 2003;29:171-177.

19. Shirahama T. Cyclooxygenase-2 expression is up-regulated in transitional cell carcinoma and its preneoplastic lesions in the human urinary bladder. Clin Cancer Res. 2000;6:2424-2430.

20. Shirahama T, Sakakura C. Overexpression of cyclooxygenase-2 in squamous cell carcinoma of the urinary bladder. Clin Cancer Res. 2001;7:558-561.

21. Kömhoff M, Guan Y, Shappell HW et al. Enhanced expression of cyclooxygenase-2 in high grade human transitional cell bladder carcinomas. Am J Pathol. 2000;157:29-35.

22. Chan G, Boyle JO, Yang EK et al. Cyclooxygenase-2 expression is up-regulated in squamous cell carcinoma of the head and neck. Cancer Res. 1999;59:991-994.

23. Maaser K, Däubler P, Barthel B et al. Oesophageal squamous cell neoplasia in head and neck cancer patients: upregulation of COX-2 during carcinogenesis. Br J Cancer. 2003;88:1217-1222.

24. Shamma A, Yamamoto H, Doki Y et al. Up-regulation of cyclooxygenase-2 in squamous carcinogenesis of the esophagus. Clin Cancer Res. 2000;6:1229-1238.

25. Li S, Miner K, Fannin R, Carl Barrett J, Davis BJ. Cyclooxygenase-1 and 2 in normal and malignant human ovarian epithelium. Gynecol Oncol. 2004;92:622-627.

26. Seo SS, Song YS, Kang DH et al. Expression of cyclooxygenase-2 in association with clinicopathological prognostic factors and molecular markers in epithelial ovarian cancer. $G y-$ necol Oncol. 2004;92:927-935. 
27. Chen YJ, Wang LS, Wang PH et al. High cyclooxygenase-2 expression in cervical adenocarcinomas. Gynecol Oncol. 2003;88:379-385.

28. Kim MH, Seo SS, Song YS et al. Expression of cyclooxygenase-1 and -2 associated with expression of VEGF in primary cervical cancer and at metastatic lymph nodes. Gynecol Oncol. 2003;90:83-90.

29. Edwards J, Mukherjee R, Munro AF, Wells AC, Almushatat A, Bartlett JM. HER2 and COX2 expression in human prostate cancer. Eur J Cancer. 2004;40:50-55.

30. Tucker ON, Dannenberg AJ, Yang EK et al. Cyclooxygenase-2 expression is up-regulated in human pancreatic cancer. Cancer Res. 1999;59:987-990.

31. Kuźbicki Ł, Sarnecka A, Chwirot BW. Expression of cyclooxygenase-2 in benign naevi and during human cutaneous melanoma progression. Melanoma Res. 2006;16: 29-36.

32. Chwirot BW, Kuźbicki Ł. Cyclooxygenase-2 (COX-2): first immunohistochemical marker distinguishing early cutaneous melanomas from benign melanocytic skin tumours. Melanoma Res. 2007;17:139-145.

33. Kuźbicki Ł, Lange D, Chwirot BW. Cyclooxygenase-2 immunohistochemistry in human melanoma: differences between results obtained with different antibodies. Melanoma Res. 2009;19:294-300.

34. Leong J, Hughes-Fulford M, Rakhlin N, Habib A, Maclouf J, Goldyne ME. Cyclooxygenases in human and mouse skin and cultured human keratinocytes: association of COX-2 expression with human keratinocyte differentiation. Exp Cell Res. 1996;224:79-87.

35. Vogt T, McClelland M, Jung B et al. Progression and NSAID-induced apoptosis in malignant melanomas are independent of cyclooxygenase II. Melanoma Res. 2001;11:587-599.

36. Putti TC, Teh M, Lee YS. Biological behavior of keratoacanthoma and squamous cell carcinoma: telomerase activity and COX-2 as potential markers. Mod Pathol. 2004; 17:468-475.

37. Kagoura M, Toyoda M, Matsui C, Morohashi M. Immunohistochemical expression of cyclooxygenase- 2 in skin cancers. J Cutan Pathol. 2001;28:298-302.

38. Nijsten T, Colpaert CG, Vermeulen PB, Harris AL, Van Marck E, Lambert J. Cyclooxygenase-2 expression and angiogenesis in squamous cell carcinoma of the skin and its precursors: a paired immunohistochemical study of 35 cases. Br J Dermatol. 2004;151:837-845.

39. Müller-Decker K, Reinerth G, Krieg P et al. Prostaglandin$\mathrm{H}$-synthase isozyme expression in normal and neoplastic human skin. Int J Cancer. 1999;82:648-656.
40. Kim KH, Park EJ, Seo YJ et al. Immunohistochemical study of cyclooxygenase-2 and p53 expression in skin tumors. J Dermatol. 2006;33:319-325.

41. An KP, Athar M, Tang $\times$ et al. Cyclooxygenase- 2 expression in murine and human nonmelanoma skin cancers: implications for therapeutic approaches. Photochem Photobiol. 2002;76:73-80.

42. Abd-El-Aleem SA, Ferguson MW, Appleton I, Bhowmick A, McCollum CN, Ireland GW. Expression of cyclooxygenase isoforms in normal human skin and chronic venous ulcers. J Pathol. 2001;195:616-623.

43. Koljonen V, Lassus P, Tukiainen E, Ristimäki A, Haglund C, Böhling T. Cyclooxygenase-2 expression in primary Merkel cell carcinoma. J Cutan Pathol. 2005;32:55-58.

44. Hsu S-M, Soban E. Color modification of diaminobenzidine (DAB) precipitation by metallic ions and its application for double immunohistochemistry. J Histochem Cytochem. 1982;30:1079-1082.

45. Scopsi L, Larsson L-I. Increased sensitivity in peroxidase immunocytochemistry. A comparative study of a number of peroxidase visualization methods employing a model system. Histochemistry. 1986;84:221-230.

46. Adler N, Tsabari C, Sulkes J, Ad-El D, Feinmesser M. Cyclooxygenase-2 expression in dermatofibroma and dermatofibrosarcoma protuberans. J Cutan Pathol. 2008;35:532-535.

47. Lebwohl M. Actinic keratosis: epidemiology and progression to squamous cell carcinoma. Br J Dermatol. 2003; 149:31-33.

48. Yantsos VA, Conrad N, Zabawski E, Cockerell CJ. Incipient intraepidermal cutaneous squamous cell carcinoma: a proposal for reclassifying and grading solar (actinic) keratoses. Semin Cutan Med Surg. 1999;18:3-14.

49. Weedon DD, Malo J, Brooks D, Williamson R. Squamous cell carcinoma arising in keratoacanthoma: a neglected phenomenon in the elderly. Am J Dermatopathol. 2010;32: $423-426$.

50. Rivers JK, Arlette J, Shear N, Guenther L, Carey W, Poulin Y. Topical treatment of actinic keratoses with $3.0 \%$ diclofenac in 2.5\% hyaluronan gel. Br J Dermatol. 2002;146: 94-100.

51. Kuźbicki Ł, Lange D, Stanek-Widera A, Chwirot BW. Cyclin-dependent Kinase 2 (CDK-2) expression in nonmelanocytic human cutaneous lesions. Appl Immunohistochem Mol Morphol. 2010;18:357-364.

52. Higashi Y, Kanekura T, Kanzaki T. Enhanced expression of cyclooxygenase (COX)-2 in human skin epidermal cancer cells: evidence for growth suppression by inhibiting COX-2 expression. Int J Cancer. 2000;86:667-671.

Submitted: 11 November, 2010

Accepted after reviews: 1 March, 2011 